\title{
Molecular diagnosis of cerebral toxoplasmosis: comparing markers that determine Toxoplasma gondii by PCR in peripheral blood from HIV-infected patients
}

\section{Authors}

Rafael Tonini Mesquita José Ernesto Vidal ${ }^{2,3}$ Vera Lucia PereiraChioccola $^{1}$

${ }^{1}$ Parasitology Laboratory, Instituto Adolfo Lutz.

${ }^{2}$ Department of Neurology, Instituto de Infectologia Emílio Ribas, SP, Brazil. ${ }^{3}$ Extension Service for Treatment of HIV/AIDS Patients, Division of Infectious and Parasitory Diseases, Hospital das Clínicas da Faculdade de Medicina da Universidade de São Paulo, Brazil.
Submitted on $03 / 05 / 2010$ Approved on $04 / 18 / 2010$

Correspondence to: Vera Lucia PereiraChioccola

Laboratório de Parasitologia, Instituto Adolfo Lutz,

Av. Dr Arnaldo, 351, $8^{\circ}$ andar São Paulo - SP - Brazil CEP: 01246-902 Phone: +55-11-30682991 Fax: +55-11-30682890 E-mail: pchioccola@ial. sp.gov.br.

This study was supported by grants from the FAPESP (Fundaçao de Amparo à Pesquisa do Estado de São Paulo, Brazil). Reference 08/09311-0.

\begin{abstract}
As cerebral toxoplasmosis is the most common cerebral focal lesion in AIDS patients, this study evaluated three PCR markers for diagnosis, since some limitations remain present, such as low parasite levels in some clinical samples. The molecular markers were B22-B23 and Tg1-Tg2 (based on the B1 gene) and Tox4-Tox5 (non-coding fragment, repeated 200-300-fold). DNA samples from 102 AIDS patients with previously known diagnosis were analyzed. The cerebral toxoplasmosis group was constituted of DNA extracted from the blood of 66 AIDS patients, which was collected before or until the third day of the therapy for toxoplasmosis. DNA from the blood of 36 AIDS patients with other neurologic opportunistic infections was used as control group. Sensitivities of B22-B23, Tg1$\mathrm{Tg} 2$, and Tox4-Tox 5 markers were of $95.5 \%, 93.9 \%$, and $89.3 \%$, respectively. In the control group, the specificities were of $97.2 \%$ (B22-B23), 88.9\% (Tg1-Tg2), and 91.7\% (Tox4-Tox5). The association of at least two markers increased the PCR sensitivity and specificity. The concordance index between two markers varied from $83.3 \%$ to $93.1 \%$. These data demonstrated that all markers evaluated here were highly sensitive for T. gondii determination, although B22-B23 has been shown to be the best. The association of two markers increases PCR sensitivity, but the procedure was more expensive and time-consuming.
\end{abstract}

Keywords: AIDS, cerebral toxoplasmosis, molecular diagnosis, PCR markers.

[Braz J Infect Dis 2010;14(4):346-350]@Elsevier Editora Ltda.

\section{INTRODUCTION}

Toxoplasma gondii is an intracellular protozoan parasite that infects all mammalian cells. Human infection is generally innocuous and asymptomatic. During the chronic phase, the parasites persist encysted in the brain and in muscles, developing life-long protective immunity against re-infection..$^{1-3}$ However, reinfections are also possible, since different $T$. gondii genotypes have been found in the same patient. ${ }^{4,5}$

Although the infection in humans is usually asymptomatic, ocular and disseminated forms of toxoplasmosis have been reported in immunocompetent patients, especially in South America. ${ }^{6,7}$ Symptomatic forms are frequent when the primary infection occurs either during pregnancy, which can result in severe neonatal malformations and ocular complications in the fetus, or in the setting of an immunodeficiency, such as AIDS. Reactivation of latent infection in AIDS patients results more frequently in cerebral toxoplasmosis and is a life-threat- ening condition without timely diagnosis and treatment. ${ }^{2,8}$

Cerebral toxoplasmosis remains a prevalent disorder of the central nervous system in developed countries, particularly among severely immunosuppressed HIV-infected patients in the absence of antibiotic prophylaxis. ${ }^{9}$ This situation is critical in most resource-limited settings, where highly active antiretroviral therapy (HAART) is not available. In Brazil, a developing country with universal and free access to HAART, cerebral toxoplasmosis still accounts for high mortality and morbidity. ${ }^{10}$

Although the definitive diagnosis of cerebral toxoplasmosis requires demonstration of tachyzoites in brain biopsy or necropsy, in clinical practice, treatment is usually initiated upon a presumptive diagnosis, which is based on clinical and radiological features. In the last decade, significant improvement has been made with the introduction of molecular diagnosis. PCR in cerebral toxoplasmosis diagnosis has been shown to be an 
important diagnostic tool. ${ }^{11-13}$ In parallel, several DNA targets were evaluated and have been used regularly in different laboratories to diagnose congenital, ocular, disseminated or cerebral toxoplasmosis. Among them, two are more frequently used due to their greater sensitivity and specificity. One is the 529-bp sequence, which has 200-300 copies in the genome of T. gondii. ${ }^{14}$ The other is the $\mathrm{B} 1$ gene, having 35 copies in the genome and conserved in different parasite strains. ${ }^{15,16}$ The sensitivity and accuracy of the target 529-bp sequence, B1 gene, and the comparison of both were largely analyzed. ${ }^{12-14,17-20}$ The majority of these studies aiming to compare sensitivities of both DNA regions were conducted in European clinical samples, but little research has been done on Brazilian samples. ${ }^{21}$ These data, combined with certain limitations in laboratorial practices, such as the low parasite levels in some clinical samples, lead us to compare two molecular markers directed at the $\mathrm{B} 1$ gene and the 529bp sequence for cerebral toxoplasmosis diagnosis.

\section{MATERIAL AND METHODS}

\section{Patients and clinical samples}

We analyzed 102 DNA samples of patients with laboratorial, clinical, and radiological diagnosis previously known. The cerebral toxoplasmosis group comprised by 66 AIDS patients with positive IgG antibodies anti T. gondii determined by indirect immunofluoresce reaction and clinical and radiological features had DNA extracted from the blood. Case definition for toxoplasmosis included the following criteria: (i) progressive neurological deficits; (ii) contrast-enhancing mass lesion(s) on computed tomography; (iii) successful response within two weeks to specific treatment. ${ }^{10,22}$ The blood samples were collected before or until the third day of the specific therapy for toxoplasmosis. ${ }^{23}$ For the control group, DNA was extracted from the blood of 36 AIDS patients with negative IgG antibodies anti T. gondii (by indirect immunofluoresce reaction) and diagnosed with other neurologic opportunistic infections, including cryptococcal meningoencephalitis, progressive multifocal leukoencephalopathy, central nervous system tuberculosis, and HIV-associated cognitive motor disorder. The ethics committees of the institutions involved approved this study.

\section{DNA purification}

The DNA of blood samples was extracted using PureLink Genomic DNA Kits (Invitrogen) according to the manufacturer's instructions. Previously, blood samples were centrifuged, washed with PBS at 2,500 g for $10 \mathrm{~min}$, and the supernatants with plasma were discarded. In order to lyse the erythrocytes, the packed cells were mixed with three times the volume of a buffer containing $150 \mathrm{mM}$ ammonium chlorate, $1 \mathrm{mM}$ potassium bicarbonate, $0.1 \mathrm{mM}$ EDTA, $\mathrm{pH}$ 7.3 , incubated for $15 \mathrm{~min}$ at room temperature under mild shaking, and centrifuged for $10 \mathrm{~min}$ at 3,000 g. The blood pellets containing only nuclei cells were digested with proteinase $\mathrm{K}(20 \mu \mathrm{g} / \mathrm{mL})$ in $50 \mathrm{mM}$ Tris- $\mathrm{HCl}$, $25 \mathrm{mM}$ EDTA, pH $8.0,2 \%$ sodium dodecyl sulfate and incubated for $30 \mathrm{~min}$ at $56{ }^{\circ} \mathrm{C}$. The DNA pellets were dissolved in ultra pure water. As a positive control, DNA was extracted from tachyzoite pellets using the same kit. DNA purity was determined by the ratio of O. D. at 260 and $280 \mathrm{~nm}$ in a NanoDrop ND100 (Thermo Scientific). ${ }^{24}$

\section{PCR}

Each reaction was performed by adding $5 \mu \mathrm{L}$ of each DNA template and 25 pmol of each primer to a final volume of $25 \mu \mathrm{L}$. The amplifications were carried out with a kit purchased from Promega (Go Taq Green Master Mix). The PCR mix $(12.5 \mu \mathrm{L})$ was composed of 1 unit of Taq DNA polymerase, $10 \mathrm{mM}$ Tris- $\mathrm{HCl}$, pH 8.5; $50 \mathrm{mM} \mathrm{KCl} ; 1.5 \mathrm{mM}$ $\mathrm{MgCl}_{2}$; and $200 \mathrm{mM}$ of each dNTP. Each amplification run contained two negative controls (ultra pure water and a negative DNA for toxoplasmosis) and one positive (DNA extracted from RH strain). After thermal cycles, PCR products were electrophoresed in 2\% agarose gel and stained with GelRed (Biotium). DNA fragments were visualized under UV illumination. The images were analyzed by a Mini Bis Gel Imager and Documentation (BioSystematica). The size of fragments was based on comparison with a 100-bp ladder.

\section{Marker selection}

We chose two markers from the $\mathrm{B} 1$ gene, since it is a 35 -fold repetitive gene with 2214 nucleotides in each repeat and is highlyconservedamong Toxoplasmastrains. ${ }^{15,16}$ The first was B22-B23 (5'AACGGGCGAGTAGCACCTGAGGAGA3' and 5'TGGGTCTACGTCGATGGCATGACAAC3') which amplified a 115-bp sequence from a specific repetitive region as target. The amplifications were made by one initial denaturation cycle for $5 \mathrm{~min}$ at $95^{\circ} \mathrm{C}, 35$ cycles of denaturation at $95^{\circ} \mathrm{C}$ for $1 \mathrm{~min}$, annealing at $62^{\circ} \mathrm{C}$ for 1 min, and extension at $72{ }^{\circ} \mathrm{C}$ for $1 \mathrm{~min}$. The procedure was completed by a final cycle extension for $5 \mathrm{~min}$. The second was Tg1-Tg2 (5'AAAAATGTGGGAATGAAAGAG3' and 5'ACGAATCAACGGAACTGTAAT3'), which amplified a 469-bp DNA fragment of the gene as target. ${ }^{25}$ The PCR products were amplified by one initial denaturation cycle for $5 \mathrm{~min}$ at $94^{\circ} \mathrm{C}, 35$ cycles of denaturation at $94{ }^{\circ} \mathrm{C}$ for $45 \mathrm{sec}$, annealing at $50^{\circ} \mathrm{C}$ for $30 \mathrm{sec}$, and extension at $72{ }^{\circ} \mathrm{C}$ for $60 \mathrm{sec}$. The procedure was completed by a final cycle extension for $7 \mathrm{~min}$. The third marker, Tox4Tox5 (5'CGCTGCAGGGAGGAAGACGAAAGTTG3' and 5'CGCTGCAGACACAGTGCATCTGGATT3'), amplified a non-coding $529 \mathrm{bp}$ fragment that is repeated 200-300- 
fold in the genome of T. gondii. ${ }^{14}$ The reaction conditions were as follows: one initial denaturation cycle for $5 \mathrm{~min}$ at $94{ }^{\circ} \mathrm{C}, 35$ cycles of denaturation at $94^{\circ} \mathrm{C}$ for $45 \mathrm{sec}$, annealing at $55^{\circ} \mathrm{C}$ for $45 \mathrm{sec}$, and extension at $72{ }^{\circ} \mathrm{C}$ for $45 \mathrm{sec}$. The procedure was completed by a final cycle extension for $5 \mathrm{~min}$. To control the course of extraction and check for PCR inhibitors, all samples were assayed using marker $\beta 1-\beta 2$ (5'ACCACCAACTTCATCCACGTTCACC3' and 5'CTTCTGACACAACTGTGTTCACTAGC3') ${ }^{26}$ that amplified a $140 \mathrm{bp}$ fragment of the human $\beta$-globulin gene. The reactions using the human marker were run simultaneously with the same temperature protocol for the B22-B23 marker and in the same PCR machine.

As an illustration, Figure 1 shows the amplified PCR products of all markers used in this study.

\section{Data analysis}

All divergent results were repeated at least twice. Sensitivity was calculated as a ratio of true positives/true positives + false negatives x 100; and specificity, as a ratio of true negatives/true negatives + false positives $\mathrm{x} 100$. The percents of concordant index (between the three markers) were calculated as the following ratio: (number of concordant results)/ (total number of samples) x 100 .

\section{RESULTS}

The marker sensitivities were analyzed using DNA extracted from the blood of 66 AIDS patients with cerebral toxoplasmosis. B22-B23, Tg1-Tg2, and Tox4-Tox5 markers amplified PCR products of 63,62 , and 58 samples, providing sensitivities of $95.5 \%, 93.9 \%$, and $89.3 \%$ respectively. The association of at least two markers increased PCR sensitivity and specificity. All positive samples had amplified products when we analyzed the results of at least one marker (B22B23/Tg1-Tg2/Tox4-Tox5). The analysis in the DNA samples of the 36 AIDS patients with other neurologic opportunistic infections and without toxoplasmosis provided the specificities of $97.2 \%, 88.9 \%$, and $91.7 \%$ for the markers B22-B23, Tg1-Tg2, and Tox4-Tox5 respectively.

The concordance index among the markers was also studied. As shown in Table 1, the overall concordance of the

Figure 1: Amplified PCR products in two different target regions of T. gondii B1 gene: B22-B23, 115 bp (A) and Tg1-Tg2, 469 bp (B). The third marker, Tox4-Tox5, $529 \mathrm{bp}(\mathrm{C})$ is a non-coding fragment in the genome of T. gondii. b1-b2 marker was used as PCR control and amplified a 140-bp fragment of human $\beta$-globulin gene (D). The DNA fragments were resolved in $2 \%$ agarose gels stained with GelRed. Lane 1, 100-bp ladder; lane 2, negative control (water); lane 3, DNA from a negative; and line 4, a positive patient for toxoplasmosis.
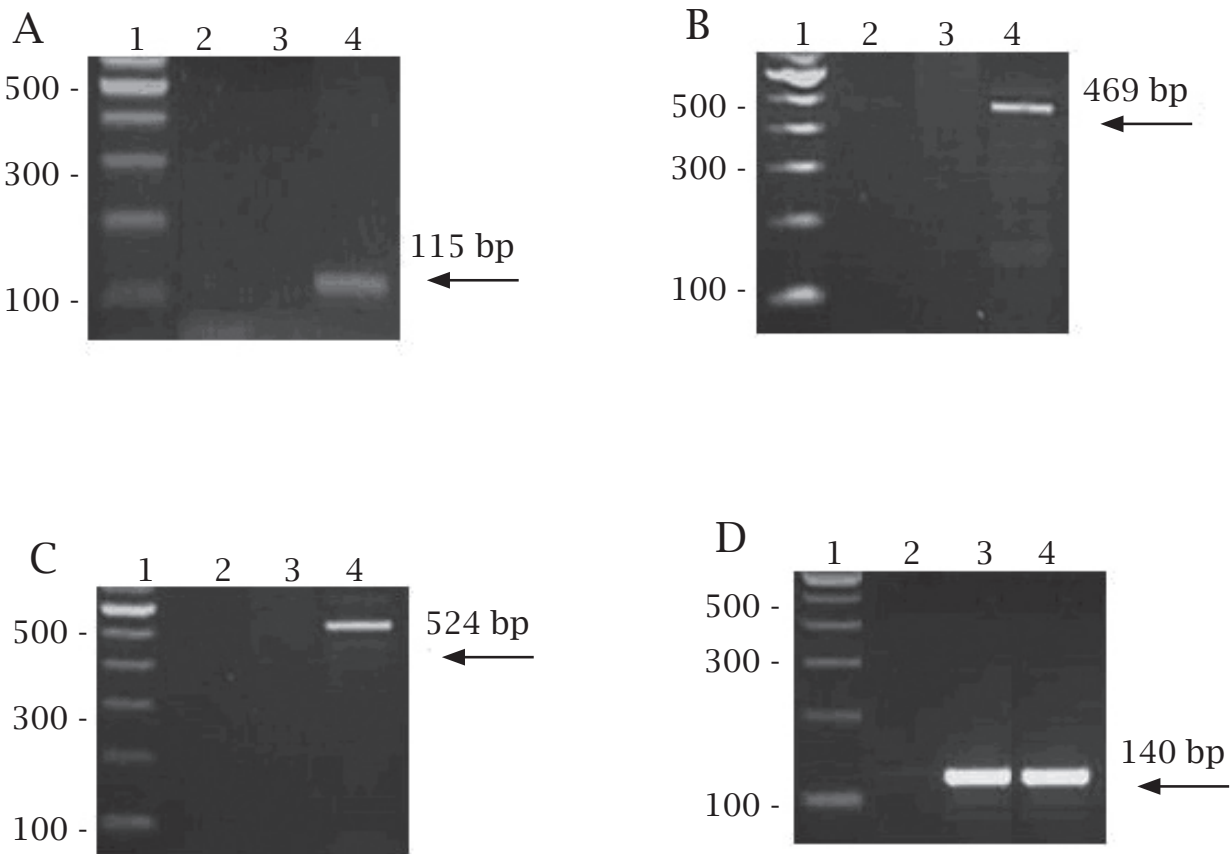
results among the three markers was of $79.4 \%$. The concordance between B22-B23 and Tox4-Tox 5 were of $83.3 \%$; between B22-B23 and Tg1-Tg2, were of 93.1\%; and Tox4-Tox5 and $\operatorname{Tg} 1-\operatorname{Tg} 2$, of $87.2 \%$.

Table 1. Concordance index between the three markers for the detection of $\mathrm{T}$. gondii in blood samples

\begin{tabular}{|c|c|c|c|}
\hline \multicolumn{4}{|c|}{ Cerebral toxoplasmosis } \\
\hline PCR markers & Concordant $^{1}$ & Discordant $^{2}$ & $\begin{array}{c}\text { Concordance } \\
\text { index }^{3}(\%)\end{array}$ \\
\hline B22-B23/Tg1-Tg2/ & & & \\
\hline Tox4-Tox 5 & 81 & 21 & $(79.4)$ \\
\hline B22-B23/Tox4-Tox5 & 85 & 17 & (83.3) \\
\hline B22-B23/Tg1-Tg2 & 95 & 07 & $(93.1)$ \\
\hline Tox4-Tox5/Tg1-Tg2 & 89 & 13 & $(87.2)$ \\
\hline
\end{tabular}

${ }^{1}$ Positive in all markers;

${ }^{2}$ Negative in at least one marker;

${ }^{3}$ Percents of concordant index were calculated as the ratio: (number of concordant results)/( total number of samples) x 100 .

\section{DISCUSSION}

Despite that, presently, cerebral toxoplasmosis has declined in AIDS patients, its occurrence still represents a determinant of poor diagnosis in the natural history of HIV-infected patients, even in the HAART era. ${ }^{9}$ Consequently, cerebral toxoplasmosis has caused high morbidity and mortality, particularly among Brazilian AIDS patients. ${ }^{10}$ Consequently, it is necessary to evaluate accurate, less invasive, and rapid diagnostic tools. Even though, the use of PCR has improved the diagnosis, some difficulties still remain in laboratorial practice. Some clinical samples have small amplified PCR products and false readings in the agarose gels due to very low parasite levels.

Different studies have shown that molecular markers that target the $\mathrm{B} 1$ gene or the repeated region of the T. gondii genome present good sensitivity for clinical samples. However, these studies were conducted by using clinical samples collected from patients living and probably infected in Europe. ${ }^{17,19,20,25,27-30}$ Here, we chose three previously described markers widely used for $T$. gondii detection, in order to determine whether the results are similar in Brazilian clinical samples. Two markers were based on the B1 gene as the target region, because it is a 35 -fold repetitive gene, highly conserved among Toxoplasma strains. The B22-B23 marker was able to amplify and detect the DNA of a single organism directly from a crude cell lysate or 10 parasites in the presence of 100,000 human leukocytes. ${ }^{15,16}$
The high sensitivity and specificity of this primer pair were previously reported compared to other $T$. gondii sequence primers. ${ }^{27}$ In addition, our group also showed its high sensitivity and specificity in cerebrospinal fluid and blood samples collected from AIDS patients and newborns. ${ }^{12,13,24}$ Tg1-Tg2 marker ${ }^{25}$ also amplifies a different region of the B1 gene in a 469 bp PCR fragment. The third marker, Tox4Tox5, which amplifies a non-coding 529 bp DNA fragment, was selected because the target region occurs in more copies than the $\mathrm{B} 1$ gene that is 35 -fold in the genome.

The sensitivity, accuracy and specificity of a diagnostic test depend on optimal working conditions. In order to improve the PCR sensitivity, the blood samples were processed rapidly, within 48 hours of collection, to prevent Taq polymerase inhibition. Many components found in biological samples (blood, urine, feces, cerebrospinal liquid) inhibit taq-polymerase, modifying PCR results. ${ }^{31}$

The plasma removal and erythrocyte lysis with a specific buffer were procedures employed before lysis of cells with nucleus for DNA extraction, in order to prevent PCR inhibitors. Furthermore, the quality of the DNA extraction was evaluated by $\beta 1-\beta 2$ marker that amplified a PCR fragment of human $\beta$-globulin gene. Positive amplifications showed that no substance present in DNA samples inhibited the reaction. All blood samples tested were collected before or until the first three days of the specific therapy. ${ }^{13}$ Previous studies reported that anti-toxoplasmic therapy decreases diagnostic sensitivity, especially if samples were collected after the first week of treatment. ${ }^{23,32}$ Finally, all divergent results between the three markers were repeated at least twice. Additionally, no amplified PCR product was observed in the negative samples, showing a high specificity for the three markers studied ( $88.9 \%$ to $97.2 \%$ ).

With respect to the sensitivities, although Tox 4 -Tox 5 targets a region repeated 200-300-fold in the genome, and the $\mathrm{B} 1$ gene is a 35-copy gene, the sensitivities of both markers from B1 gene (B22-B23 and Tg1-Tg2) were of 93.9\% to $95.9 \%$, while the sensitivity of the Tox4-Tox 5 was of $89.3 \%$. These data are consistent with a recent Brazilian study conducted by Okay et al. ${ }^{21}$ In addition, previous studies have also shown high sensitivity and specificity for markers designed to amplify different regions of the B1 gene in clinical samples. ${ }^{13,18,19,25,27,29}$ Other studies conducted using European clinical samples aiming to compare sensitivities of both DNA regions have shown that those from repeat regions were more sensitive than those from the B1 gene. ${ }^{14,20,28,30}$

The concordance index between two markers varied from $83.3 \%$ to $93.1 \%$. These data demonstrated that all markers evaluated here were highly sensitive for $T$. gondii detection, although B22-B23 has been shown to be the best. The association of two markers increases the PCR sensitivity, but the procedure was more expensive and time-consuming. 


\section{REFERENCES}

1. Dubey JP. Advances in the life cycle of Toxoplasma gondii. Int J Parasitol 1998; 7:1019-24.

2. Montoya JG, Liesenfeld O. Toxoplasmosis. Lancet 2004; 363:1965-76.

3. Hill DE, Chirukandoth S, Dubey JP. Biology and epidemiology of Toxoplasma gondii in man and animals. Anim Health Res Rev 2005; 6:41-61.

4. Ferreira IMR, Vidal JE, Costa-Silva TA et al. Toxoplasma gondii: genotyping of strains from Brazilian AIDS patients with cerebral toxoplasmosis by multilocus PCR-RFLP markers. Exp Parasitol 2008; 118:221-7.

5. Elbez-Rubinstein A, Ajzenberg D, Dardé ML et al. Congenital toxoplasmosis and reinfection during pregnancy: case report, strain characterization, experimental model of reinfection, and review. J Infect Dis 2009; 199:280-5.

6. Glasner PD, Silveira C, Kruszon-Moran D et al. An unusually high prevalence of ocular toxoplasmosis in Southern Brazil. Am J Ophthalmol 1992; 114:136-44.

7. Carme B, Bissuel F, Ajzenberg D et al. Severe acquired toxoplasmosis in immunocompetent adult patients in French Guiana. J Clin Microbiol 2002; 40:4037-44.

8. Pereira-Chioccola VL, Vidal JE, Su C. Toxoplasma gondii infection and cerebral toxoplasmosis in HIV-infected patients. Future Microbiol 2009; 4:1363-79.

9. Antinori A, Larussa D, Cingolani A et al. Prevalence, associated factors, and prognostic determinants of AIDS-related toxoplasmic encephalitis in the era of advanced highly active antiretroviral therapy. Clin Infect Dis 2004; 39:1681-91.

10. Vidal JE, Hernandez AV, Penalva de Oliveira AC et al. Cerebral toxoplasmosis in HIV-positive patients in Brazil: clinical features and predictors of treatment response in the HAART era. AIDS Patient Care STDS 2005; 19:840-8.

11. Bretagne S. Molecular diagnostics in clinical parasitology and mycology: limits of the current polymerase chain reaction (PCR) assays and interest of the real-time PCR assays. Clin Microbiol Infect 2003; 9:505-11.

12. Vidal JE, Colombo FA, Penalva de Oliveira AC et al. PCR assay using cerebrospinal fluid for diagnosis of cerebral toxoplasmosis in Brazilian AIDS patients. J Clin Microbiol 2004; 42:4765-8.

13. Colombo FA, Vidal JE, Penalva de Oliveira AC et al. Diagnosis of cerebral toxoplasmosis in AIDS patients in Brazil: importance of molecular and immunological methods using peripheral blood samples. J Clin Microbiol 2005; 43:5044-7.

14. Homan WL, Vercammenb M, De Braekeleer J, Verschueren H. Identification of a 200-to 300-fold repetitive 529 bp DNA fragment in Toxoplasma gondii, and its use for diagnostic and quantitative PCR. Inter J Parasitol 2000; 30:69-75.

15. Burg JL, Grover CM, Pouletty P, Boothroyd JC. Direct and sensitive detection of a pathogenic protozoan, Toxoplasma gondii, by polymerase chain reaction. J Clin Microbiol 1989; 27:1787-92.

16. Grover CM, Thulliez P, Remington JS, Boothroyd JC. Rapid prenatal diagnosis of congenital Toxoplasma infection by using polymerase chain reaction and amniotic fluid. J Clin Microbiol 1990; 28:2297-301.
17. Montoya JG, Parmley S, Liesenfield O et al. Use of the polymerase chain reaction for diagnosis of ocular toxoplasmosis. Ophthalmology 1999; 106:1554-63.

18. Jones CD, Okhravi N, Adamson $\mathrm{P}$ et al. Comparison of PCR detection methods for B1, P30, and 18S rDNA genes of Toxoplasma gondii in aqueous humor. Invest Ophthalmol Vis Sci 2000; 41:634-44.

19. Calderaro A, Piccolo G, Gorrini C et al. Comparison between two Real-time PCR assays and a nested-PCR for the detection of Toxoplasma gondii. Acta Biomed 2006; 77:75-80.

20. Edvinsson B, Lappalainen M, Evengård B, ESCMID Study Group for Toxoplasmosis. Real-time PCR targeting a 529-bp repeat element for diagnosis of toxoplasmosis. Clin Microbiol Infect 2006; 12:131-6.

21. Okay TS, Yamamoto L, Oliveira LC et al. Significant performance variation among PCR systems in diagnosing congenital toxoplasmosis in São Paulo, Brazil: analysis of 467 amniotic fluid samples. Clinics 2009; 641:171-6.

22. Portegies P, Solod L, Cinque $\mathrm{P}$ et al. Guidelines for the diagnosis and management of neurological complications of HIV infection. Eur J Neurol 2004; 11:297-304.

23. Cingolani A, De Luca A, Ammassari A et al. PCR detection of Toxoplasma gondii DNA in CSF for the differential diagnosis of AIDS-related focal brain lesions. J Med Microbiol 1996; 45:472-6.

24. Mesquita RT, Ziegler AP, Hiramoto RM, Vidal JE, PereiraChioccola VL. Real-time quantitative PCR in cerebral toxoplasmosis diagnosis of Brazilian human immunodeficiency virus-infected patients. J Med Microbiol 2010; 59:641-7.

25. Jalal S, Nord CE, Lappalainen M, Evengard B. Rapid and sensitive diagnosis of Toxoplasma gondii infections by PCR. Clin Microbiol Infect 2004; 10:937-9.

26. Lee CN, Cavanagh HM, Lo ST, Ng CS. Human papillomavirus infection in non-neoplastic uterine cervical disease in Hong Kong. Brit. J. Biomed. Sci. 2001; 58:85-91.

27. Chabbert E, Lachaud L, Crobu L, Bastien P. Comparison of two widely used PCR primer systems for detection of Toxoplasma in amniotic fluid, blood and tissues. J Clin Microbiol 2004; 42:1719-22.

28. Cassaing S, Bessières MH, Berry A et al. Comparison between two amplification sets for molecular diagnosis of toxoplasmosis by real-time PCR. J Clin Microbiol 2006; 44:720-4.

29. Costa JM, Pautas C, Ernault P et al. Real-time PCR for diagnosis and follow-up of Toxoplasma reactivation after allogeneic stem cell transplantation using fluorescence resonance energy transfer hybridization probes. J Clin Microbiol 2000; 38:2929-32.

30. Reischl U, Bretagne S, Krüger D et al. Comparison of two DNA targets for the diagnosis of toxoplasmosis by real-time PCR using fluorescence resonance energy transfer hybridization probes. BMC Infect Dis 2003; 3:7-9.

31. Al-Soud WA, Radstrom P. Capacity of nine thermostable DNA polymerases to mediate DNA amplification in the presence of PCR-inhibiting samples. Appl Environ Microbiol 1998; 64:3748-53.

32. Gianotti N, Cinque P, Castagna A et al. Diagnosis of toxoplasmic encephalitis in HIV-infected patients. AIDS 1997; 11:1529-30. 